\title{
Neuroscience and Psychiatry Relational Memory as a Possible Neurocognitive Marker of Schizophrenia
}

Martin Lepage, PhD; Colin Hawco, PhD; Michael Bodnar, PhD

Long-term or episodic memory can be understood along several dimensions, including verbal vs abstract, spatial vs nonspatial, and item vs relational memory. The latter has represented a field of growing interest over the past 15 years, particularly in cognitive neuroscience and neuroimaging. In basic terms, item memory

Related article page 909

is a metric related to a single piece of information, whereas relational memory involves forming a relationship between 2 or more pieces of information. Relational memory can occur within many contexts such as linking multiple objects together (eg, "I saw $x$ with $y$. .) or within a spatial or temporal context (eg, "I saw $x$ at the park in the afternoon."). However, relational memory cannot be considered successful in the absence of item memory (eg, "I saw someone at the park."). Relational memory requires more elaborate processing to create associations that can later be recalled together. By consequence, memory performance for individual items can be improved with deep semantic encoding (eg, combining items into groups) as opposed to shallow encoding (eg, remembering items individually). Not surprisingly, forming relationships between items is easiest when items share semantic features such as category (eg, hammer and screwdriver are tools).

The foundation of understanding the neural basis of item and relational memory was based on lesion studies in which relational memory was selectively impaired, despite relatively preserved item memory following hippocampal or prefrontal damage. Now a technique, such as functional magnetic resonance imaging (fMRI), makes it possible to continue advancing our knowledge and to further our understanding of a specific cognitive process (eg, item vs relational memory) at the neuronal level. It also allows us to explore specific cognitive deficits in psychiatric research with relative ease.

\section{Measuring Relational Memory in the Brain}

Episodic memory processes occur and can be measured during the 2 critical phases of (1) encoding, when information is initially processed, and (2) retrieval (or recognition), when information is brought back into active cognitive processing. It can be difficult to truly separate these processes because correct retrieval requires successful encoding, but the mark of successful encoding is correct retrieval. In the cognitive neurosciences, a powerful tool used to help distinguish successful from unsuccessful encoding is the subsequent memory effect. ssentially, encoding is deemed successful for each correct response given during a postencoding memory retrieval task, and vice versa.

Multiple fMRI studies have used this approach to examine relational memory, with the results showing a division of function in the prefrontal cortex (PFC). ${ }^{1}$ The ventrolateral PFC is involved in general memory formation, whereas the dorsolateral PFC is specifically involved in relational memory encoding. Most important, this effect was present even when task demands were equalized across item and relational encoding, ${ }^{1}$ suggesting that dorsolateral PFC activity is specific to relational encoding and not a by-product of greater task demands. The hippocampus is well established as another critical region in relational memory processes. Multiple fMRI studies have demonstrated increased activation during encoding and retrieval, with more consistent activation during retrieval. ${ }^{2}$ This is not to say that the hippocampus does not have a role in encoding but instead that it may be active during successful and unsuccessful encoding. The success of relational memory encoding may just be more dependent on cortical structures such as the PFC.

Relational memory appears to be dependent on a distinct neural network. In a basic sense, the PFC and medial temporal lobe (MTL) display nonspecific activity during general memory processes; however, when engaged in a relational memory process, the hippocampus and the dorsolateral PFC are also recruited. ${ }^{1,3}$ In addition, any dysfunction in these specific subregions should lead to poorer relational memory, leaving item memory relatively intact.

\section{Relational Memory Deficits in Schizophrenia}

and Their Implications

In line with the original lesion studies, research involving schizophrenia has also found relational memory to be selectively impaired despite item memory being relatively preserved. These studies documented greater impairment in recognition memory tasks involving relational memory relative to item recognition, suggesting that processes inherent to relational memory could be more affected than others. For instance, work from our laboratory examined item recognition (both images old or new) vs relational recognition (image pairs rearranged or intact). ${ }^{4}$ Indeed, individuals with schizophrenia compared with healthy control participants demonstrated significantly worse relational recognition ability and normal item recognition ability. This result suggested that in schizophrenia the ability to associate separate aspects of events into a cohesive and distinctive whole may be impaired.

With the neural foundation for relational memory understood to involve the PFC and MTL, fMRI investigations using an innovative behavioral task (eg, the study by Ragland et al ${ }^{5}$ published in this issue) are making it possible to enhance our understanding of the neural correlates of the selective memory impairments observed in schizophrenia. The Relational and Item-Specific (RiSE) task by Ragland et al cleverly examines the encoding of image pairs as a function of 2 orienting tasks promoting item encoding ("Is either object living?") or relational encoding ("Can one object fit inside the other?"). For item recognition, participants indicated whether individual images were previously studied (old) or never studied (new). For associative recognition, participants indicated whether object pairs were unchanged (ie, image pairs encoded together) or changed (ie, image pairs encoded separately). All participants completed 1 encoding and 2 retrieval fMRI runs. For encoding, the schizophrenia 
group compared with healthy controls exhibited greater difficulties with relational encoding relative to item encoding and showed decreased activation in the dorsolateral PFC and normal activation in the ventrolateral PFC. For recognition, the schizophrenia group compared with healthy controls exhibited poorer associative recognition and poorer item recognition of images that were relationally encoded. The fMRI result revealed reduced hippocampal activity during successful item recognition of images that were relationally encoded. Consistent with the current literature, a picture is emerging with findings supporting that the dorsolateral PFC is pertinent for relational encoding and that reduced activity in specific regions of the PFC and MTL may be associated with the selective relational memory impairment observed in schizophrenia.

Studies measuring deficient memory processes in schizophrenia and their underlying neural architecture are important for several reasons. Considering the significant association between memory impairment and poorer outcome in schizophrenia, ${ }^{6}$ the behavioral and neurobiological results from these studies provide novel targets for future pharmacological, psychosocial, or neurostimulation interventions. Studies such as that by Ragland et al ${ }^{5}$ and others (eg, see the study by Bodnar et $\mathrm{al}^{7}$ ) bring us closer toward translating psychiatric neuroimaging into clinical practice. ${ }^{8}$ They also make it possible to examine the interplay between such cognitive deficits and the symptoms of schizophrenia, ultimately contributing to a new understanding of the pathophysiology of this disorder.

Several studies have examined the association between the symptoms of schizophrenia and relational memory deficits. For example, Hawco et al ${ }^{9}$ examined relational (source) memory whereby participants navigated through a 3-dimensional virtual city, leading to encounters consisting of a person and an object at a specific location. During recognition, the individuals with schizophrenia showed a significant deficit in recognizing "item with person" and demonstrated a reduced pattern of neural activity relative to controls in several brain regions, including the PFC. Positive correlations with hallucination proneness were observed with activity in the left frontal and right middle temporal cortices and cerebellum, regions known to activate during active hallucinations. This supported previous models relating hallucinations and deficits in relational memory, as well as a tendency to misattribute self-generated stimuli to an external source.

Memory deficits in schizophrenia may be strongest for relational memory and, as implied above, may stem from failing to spontaneously use effective memory strategies and not activating extended memory networks during encoding or retrieval. This knowledge is paving the way forward to create novel interventions specifically targeted at these selective deficient memory processes. For example, Guimond and Lepage ${ }^{10}$ have recently developed and tested a brief intervention designed to improve spontaneous use of semantic encoding strategies. Individuals with schizophrenia and a relational memory deficit were offered a 2-hour cognitive remediation training course. This course taught participants to use encoding strategies involving the organization of information as a function of semantic categories, with exercises provided to promote spontaneous use of such strategies. Following training, participants demonstrated significantly improved performance on multiple memory measures. Therefore, this specific memory deficit can be reduced when patients are specifically cued to engage in deeper task processing or to use alternative memory strategies such as building semantic relationships or imagining the objects interacting.

\section{Conclusions}

In summary and as alluded to by Ragland et al, ${ }^{5}$ relational memory deficits are becoming recognized as a reliable cognitive marker of schizophrenia and neuronal activity abnormalities as a potential biomarker of such an impairment. A point to consider from our laboratory's study ${ }^{10}$ of specialized cognitive remediation is the presence of individual differences of relational memory deficits among individuals with schizophrenia. Therefore, to better understand these individual differences, it is vital to determine the onset of this deficit among populations at risk and with early psychosis. In addition, these studies will help determine if this relational memory deficit is general to all psychoses or is specific to schizophrenia.

\section{ARTICLE INFORMATION}

Author Affiliations: Department of Psychiatry, McGill University, Montreal, Quebec, Canada (Lepage); Department of Psychology, McGill University, Montreal, Quebec, Canada (Lepage, Bodnar); Douglas Mental Health University Institute, Montreal, Quebec, Canada (Lepage, Bodnar); Temerty Centre for Therapeutic Brain Intervention, Centre for Addiction and Mental Health, Toronto, Ontario, Canada (Hawco).

Corresponding Author: Martin Lepage, PhD, Douglas Mental Health University Institute, 6875 LaSalle Blvd, Montreal, QC H4H 1R3, Canada (martin.lepage@mcgill.ca).

Accepted for Publication: May 13, 2015.

Published Online: July 22, 2015.

doi:10.1001/jamapsychiatry.2015.0488.

\section{Conflict of Interest Disclosures: None reported.}

Funding/Support: A portion of the work presented in this article was supported by grants 53280 and 68961 from the Canadian Institutes of Health Research. Dr Lepage is supported by a James McGill Professorship from McGill University, and Dr Hawco is supported by a Banting Fellowship from the Canadian Institutes of Health Research.

Role of Funder/Sponsor: The funding sources had no role in the design and conduct of the study; collection, management, analysis, and interpretation of the data; preparation, review, or approval of the manuscript; and decision to submit the manuscript for publication.

\section{REFERENCES}

1. Blumenfeld RS, Parks CM, Yonelinas AP, Ranganath C. Putting the pieces together: the role of dorsolateral prefrontal cortex in relational memory encoding. J Cogn Neurosci. 2011;23(1):257-265.

2. Davachi L. Item, context and relational episodic encoding in humans. Curr Opin Neurobiol. 2006;16 (6):693-700.

3. Konkel A, Cohen NJ. Relational memory and the hippocampus. Front Neurosci. 2009;3(2):166-174.

4. Luck D, Montoya A, Menear M, Achim AM, Lal S, Lepage M. Selective pair recognition memory impairment with no response bias in schizophrenia. Psychiatry Res. 2009;169(1):39-42.

5. Ragland JD, Ranganath C, Harms MP, et al. Functional and neuroanatomic specificity of episodic memory dysfunction in schizophrenia: a functional magnetic resonance imaging study of the relational and item-specific encoding task [published online July 22, 2015]. JAMA Psychiatry. doi:10.1001/jamapsychiatry.2015.0276.

6. Lepage M, Bodnar M, Bowie CR. Neurocognition: clinical and functional outcomes in schizophrenia. Can J Psychiatry. 2014;59(1):5-12.

7. Bodnar M, Achim AM, Malla AK, Joober R, Benoit A, Lepage M. Functional magnetic resonance imaging correlates of memory encoding in relation to achieving remission in first-episode schizophrenia. $\mathrm{Br}$ J Psychiatry. 2012;200(4):300-307.

8. Borgwardt S, Fusar-Poli P. Third-generation neuroimaging in early schizophrenia. Br J Psychiatry. 2012;200(4):270-272.

9. Hawco C, Buchy L, Bodnar M, et al. Source retrieval is not properly differentiated from object retrieval in early schizophrenia. Neuroimage Clin. 2015;7:336-346.

10. Guimond S, Lepage M. Training of self-initiation of semantic encoding strategies in schizophrenia. Neuropsychol Rehabil. In press. 